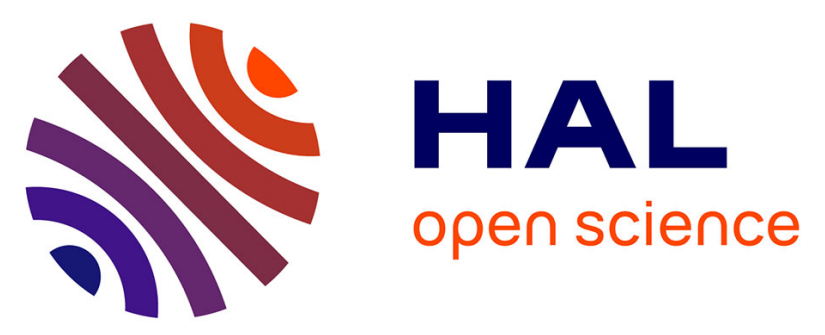

\title{
A novel stationary phase based on amino derivatized nanotubes for HPLC separations: theoretical and practical aspects \\ C. André, T. Gharbi, Y.-C. Guillaume
}

\section{- To cite this version:}

C. André, T. Gharbi, Y.-C. Guillaume. A novel stationary phase based on amino derivatized nanotubes for HPLC separations: theoretical and practical aspects. Journal of Separation Science, 2009, 32 (10), pp.1757-1764. 10.1002/jssc.200800683 . hal-00455088

\section{HAL Id: hal-00455088 \\ https://hal.science/hal-00455088}

Submitted on 26 Apr 2021

HAL is a multi-disciplinary open access archive for the deposit and dissemination of scientific research documents, whether they are published or not. The documents may come from teaching and research institutions in France or abroad, or from public or private research centers.
L'archive ouverte pluridisciplinaire HAL, est destinée au dépôt et à la diffusion de documents scientifiques de niveau recherche, publiés ou non, émanant des établissements d'enseignement et de recherche français ou étrangers, des laboratoires publics ou privés.

\section{(ㄷ)(1)}

Distributed under a Creative Commons Attribution| 4.0 International License 


\title{
A novel stationary phase based on amino derivatized nanotubes for HPLC separations: Theoretical and practical aspects
}

\author{
Claire André ${ }^{1}$, Tijani Gharbi ${ }^{2}$, Yves-Claude Guillaume ${ }^{1}$ \\ 'Equipe des Sciences Séparatives Biologique et Pharmaceutiques (EA4267), Laboratoire de Chimie Analytique, Faculté de Médecine \\ Pharmacie, Besançon, France
}

${ }^{2}$ UMR6174 Franche-Comté électronique mécanique thermique et optique, Besançon, France

\begin{abstract}
A novel column based on silica containing immobilized carbon nanotubes (CNTs) was developed and evaluated in terms of its binding efficiency and resolution. First, CNT functionalized with amino groups $\left(\mathrm{CNT}^{\left.-\mathrm{NH}_{2}\right)}\right.$ were prepared via chemical mod-ification of carboxylic groups introduced on the CNT surface. Secondly the covalent immobilization of CNT-NH$H_{2}$ was carried out by using glutardialdehyde activating agent on aminopropyl (AP)-silica surface. This CNT stationary phase was applied to the HPLC separation of two molecule series, i.e. polychlorinated biphenyl (PCB) iso-mers with different degrees of substitution in the ortho-position (non-ortho to tetra-ortho substituted) and terpenes (linalool, geraniol, thymol, $\alpha$-terpineol). The reten-tion behavior of these solute molecules was measured under isocratic conditions with different mobile phase compositions, ranging from $0.05-0.70 \mathrm{v} / \mathrm{v}$ of toluene in cyclohexane. The retention factors of the solute molecule do not depend linearly on the toluene fraction but follow a quadratic relationship. This CNT stationary phase was a very useful column for the separation of PCB congeners and terpenes. It was demonstrated that a planar conformation of the solute molecule enhanced the sol-ute retention on this CNT stationary phase. As well, a quantitative structure rela-tionship derived, demonstrated the significant input to retention was due to the structurally selective dipole-dipole and charge transfer interactions with the solutes. These results were compared with those obtained on the AP stationary phase. The proposed CNT stationary phase for the separation possess distinctive and interesting retentive properties, and chemometric analysis of retention data of appropriate designed series of test solutes appears to be a convenient, objective and quantitative method to prove a new phase specificity.
\end{abstract}

Keywords: Carbon nanotubes / HPLC / Stationary phase / Quantitative structure-retention relationships

\section{Introduction}

Nowadays, as technology progressed, novel nanomaterials with properties that are unique to the nanometer scale increased their use in analytical sciences [1-3]. The combined promise of their unique behavior at the nano-

Correspondence: Professor Yves-Claude Guillaume, Equipe des Sciences Séparatives Biologique et Pharmaceutiques (2SBP)EA4267, Laboratoire de Chimie Analytique, Faculté de Médecine Pharmacie, CHU Jean Minjoz Besançon, Place Saint Jacques, 25030 Besançon Cedex, France

E-mail: yves.guillaume@univ-fcomte.fr

Fax: +33-38-166-5655

Abbreviations: AP, aminopropyl; CNT, carbon nanotube; CNT$\mathrm{NH}_{2}$, CNT with amino groups; LFER, linear free-energy relationships; PCB, polychlorinated biphenyl; XPS, X-ray photoelectron spectroscopy meter scale with their obvious utility in miniaturization provides the main drive for their use. In recent years, a large number of allotropic carbon nanoparticles have been described in the literature, including nanodiamonds [4], fullerene $\mathrm{C}_{60}$ [5], nano-onions [6], carbon nanofibers [7], nanotube rings [8], and carbon nanotubes (CNT) [9] and single-walled CNTs (SWCNTs) [10]. Effective amino-functionalization of CNTs were used for reinforcing epoxy polymer composite [11]. As well, CNTs were used as stationary phase in GC [12-14], HPLC [15-17] and in electrochromatography [18]. The non-covalent interaction established between the analyte and these nanostructured materials includes electrostatic interactions (e.g. dipole-dipole), hydrogen bonds, $\pi$ - $\pi$ stacking, dispersion forces, dative bonds and the hydrophobic effect. The presence of supramolecular aggregates allows 
the possibility of incorporating one or more of these interactions. The combination of two or more, similar or different interactions, increases the selectivity and the stability of the system. Structure chromatographic retention relationships are amongst the most widely studied linear free-energy relationships (LFER). The equation of LFER expresses the relationship between the retention parameters determined for a representative series of analytes in a given separation system (i.e. retention factor) and the solute parameters (i.e. structural descriptors) [19-21]. This paper will first examine the preparation of a silica for the immobilization of CNT functionalized with amino groups $\left(\mathrm{CNT}-\mathrm{NH}_{2}\right)$ via chemical modification of carboxylic groups introduced on the CNT surface. The resulting packed silica column containing immobilized CNT will be evaluated in terms of its retention and selectivity for two molecules series: aromatic compounds, i.e polychlorinated biphenyls (PCBs) and terpenes, i.e. linalool, geraniol, thymol and $\alpha$-terpineol. The molecular retention mechanism (revealing the types of interactions responsible for the retention) on this novel column stationary phase was analyzed as well and compared with the one obtained on the aminopropyl (AP) stationary phase.

\section{Experimental}

\subsection{Reagents}

Water was obtained from an Elgastat water purification system (Odil, Talant, France) fitted with a reverse-osmosis cartridge. All organic solvents and test solutes were of analytical grade. Toluene, cyclohexane, and 3-aminopropyl triethoxysilane were obtained from Sigma Aldrich (Saint Quentin, France). Sodium borohydride and monoethanolamine were purchased from Sigma Aldrich (Paris, France). SWCNTs (sample purity: 50-70 vol\%, as determined by Raman Spectroscopy and SEM. Contains residual catalyst impurities (Ni, Y). Tubes occurred in bundle of lengths of $20 \mu \mathrm{m}$. Individual tube length has not been determined) were purchased from Aldrich-Co. PCBs standards were obtained from Interchim (Montluçon, France). Terpenes, i.e. thymol, linalool, geraniol and $\alpha$-terpineol were purchased from SigmaAldrich. The molecular structure of PCBs and terpenes were given in Fig. 1. A series of test solutes for quantitative structure-retention relationships studies formed 21 well-identified chemicals of various origin.

\subsection{Equipment}

The HPLC system for these measurements consisted of a Merck Hitachi pump L7100 (Nogent sur Marne, France), an Interchim Rheodyne injection valve model 7125 and a $20 \mu \mathrm{L}$ sample loop injection, and a Merck L4500 diode

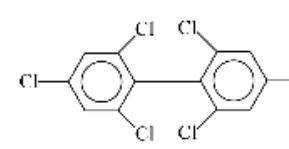

PC'B1

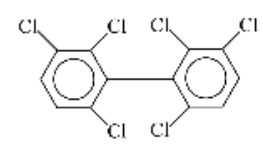

$\mathrm{PCB} 2$

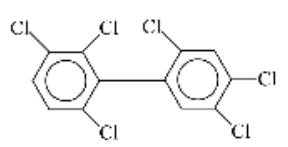

PCB3

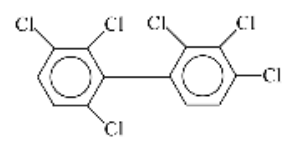

P'CB4

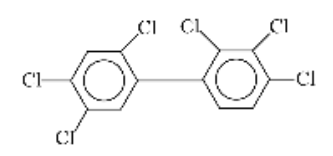

PCB5

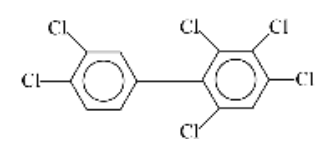

PCB6
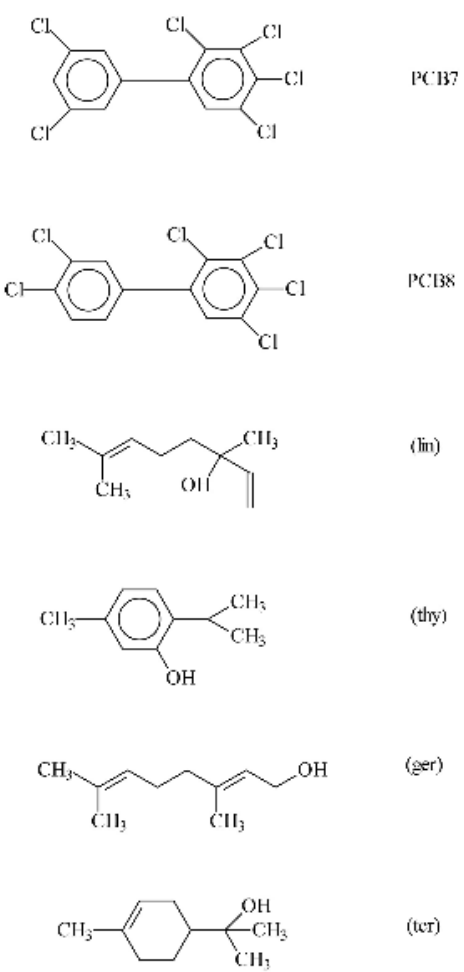

Figure 1. Molecular structures of PCBs (PCB1, PCB2, PCB3, PCB4, PCB5, PCB6, PCB7, PCB8) and terpenes (linalool (lin), thymol (thy), geraniol (ger), $\alpha$-terpineol (ter)). 
array detector. The column temperature was controlled with an Interchim oven. The Silica Kromasil column (3.5 $\mu \mathrm{m}$ particle size, $50 \times 4.6 \mathrm{~mm}$ column size) was furnished by Interchim. The $50 \times 4.6 \mathrm{~mm}$ CNT-HPLC column (CNT immobilization process) was thermostated using an Interchim oven $\mathrm{TM} \mathrm{N}^{\circ} 701$. The mobile phase was a cyclohexane-toluene mixture.

\subsection{Method}

\subsubsection{SWNTs-COOH preparation}

SWNTs-COOH were prepared as follows [22, 23]: $50 \mathrm{mg}$ of SWNTs was suspended in $200 \mathrm{~mL}$ of a 3:1 mixture of concentrated $\mathrm{H}_{2} \mathrm{SO}_{4} / \mathrm{HNO}_{3}$ in a $500 \mathrm{~mL}$ flask and sonicated in a water bath for $24 \mathrm{~h}$ at $35-40^{\circ} \mathrm{C}$. The resultant suspension was then diluted with $1000 \mathrm{~mL}$ of water and sedimentated for 3 days. The shortened SWNTs-COOH were collected by centrifugation, washed with $20 \mathrm{~mL}$ of water, and dried.

\subsubsection{Reduction of oxidized SWCNT [24]}

Twenty milligrams of SWCNT-COOH was dissolved in anhydrous THF and then $10 \mathrm{mg}$ of phtalamide was added followed by $2 \mathrm{mg}$ of diethylazodicarboxylate coupling agent. After treatment with TFA the resulting product, with aminomethyl groups attached to the SWCNT, was dried at $80^{\circ} \mathrm{C}$.

\subsubsection{CNT immobilization process}

The in situ process, which consists of the attachment of CNT-NH2 directly in pre-packed columns, was used to immobilize CNT to create this CNT column. This covalent immobilization of CNT was carried out by using glutardialdehyde activating agent on AP-silica Kromasil column. The silanol groups of the Kromasil silica stationary phase ( $3.5 \mu \mathrm{m}$ particle size) contained in a stainless column $(50 \times 4.6 \mathrm{~mm}$ id $)(50 \mathrm{~mm}$ in length containing approximatively $0.25 \mathrm{~g}$ silica) are silylated with 3-AP triethoxysilane. For this, the surface modification of the dried silica Kromasil column was carried out with a solution of $12 \mu \mathrm{mol} / \mathrm{m}^{2}$ (calculated based on $323 \mathrm{~m}^{2} / \mathrm{g}$ specific surface area of the unmodified Kromasil) of 3-AP triethoxysilane in dry toluene by pumping the reaction solution through the column at a volumetric flow rate of $0.2 \mathrm{~mL} / \mathrm{min}$ for $24 \mathrm{~h}$. During the reaction, the Kromasil column was stored inside a HPLC oven at $70^{\circ} \mathrm{C}$. In order to remove the unreacted amount of 3-AP triethoxysilane, the column was then flushed for an additional $2 \mathrm{~h}$ with toluene at a volumetric flow-rate of $0.5 \mathrm{~mL} / \mathrm{min}$. The column was then washed at the same flow-rate first with $60 \mathrm{~mL}$ ACN and finally with $60 \mathrm{~mL}$ methanol/phosphate buffer (pH 7) (0.15:0.85 v/v). The obtained AP-silica Kromasil column was activated by recycling a $5 \%$ aqueous solution of glutardialdehyde for $10 \mathrm{~h}$ at $0.5 \mathrm{~mL} / \mathrm{min}$ in the dark. CNT-NH2 is then immobilized to the silica gel by imine formation between the aldehyde group and one or more amino groups in the CNT-NH$H_{2}$. For this, a solution of $2 \mathrm{~g} / \mathrm{L}$ of $\mathrm{CNT}^{-\mathrm{NH}_{2}}$ in the methanol/phosphate buffer (pH 7.0) (0.15:0.85 v/v) was then pumped through the support at $0.5 \mathrm{~mL} / \mathrm{min}$ for $16 \mathrm{~h}$, flushing and back-flushing every $15 \mathrm{~h}$ during the first hour, every 30 min during the following $3 \mathrm{~h}$. After immobilization, a subsequent reduction of the resulting double bonds was performed with sodium borohydride. For this, the Schiff bases were reduced by recycling a cyanoborohydride solution in phosphate buffer (50 mM, pH 6.0) for $10 \mathrm{~h}$ in the dark. The column was then rinsed for $1 \mathrm{~h}$ with the methanol/ phosphate buffer (pH 7.0) (0.15:0.85 v/v) at a flow-rate of $0.5 \mathrm{~mL} / \mathrm{min}$. Finally, the unreacted aldehyde groups were capped with monoethanolamine. For this, the column was flushed with $0.2 \mathrm{M}$ monoethanolamine solution in phosphate buffer (50 mM, pH 6.0) at room temperature and finally washed and conditioned with this same buffer. The relative loading of the column material can be assessed from the carbon content determined with an elemental analysis. For this once, four fractions of the stationary phase were removed from the head to the end of the column.

\subsubsection{Measurements of physicochemical properties of stationary phase materials}

The specific surface area of the stationary phase was determined by the low-temperature nitrogen adsorptiondesorption method at $-197.5^{\circ} \mathrm{C}$ using a model 1800 Sorptomatic instrument (Carlo-Erba, Milan, Italy). The degree of coverage of silica support surface with bonded ligands was calculated by using the equations described in [25] from carbon content determined by elemental analysis with a CHN analyzer, Model 240 (Perkin Elmer, Norwalk, CT).

\subsubsection{X-ray photoelectron spectroscopy (XPS)}

XPS was used to verify the SWCNT chemical functionalization process. The chemically modified nanotubes were heated in a vacuum oven at $80^{\circ} \mathrm{C}$ overnight prior XPS characterization. XPS samples are prepared by placing the CNT sample on carbon conductive tape on the sample holder.

\subsubsection{Chemometric comparison of separation properties of the CNT and AP phases. Test solutes and their structural descriptors}

The test solutes were subjected to molecular modelling by the HyperChem package with the extension, ChemPlus (HyperCube, Waterloo, Canada). In effect, a number of quantum chemical and standard additive/constitutive structural descriptors have been generated. The descriptors which were found to be significant in the correlation 
Table 1. Test solutes and their chemistry structural descriptors.

\begin{tabular}{lrlr}
\hline Solute & \multicolumn{1}{l}{ Е номо } & $\mu$ & \multicolumn{1}{l}{$\mathrm{M}$} \\
\hline 1,3,5-Triisopropylbenzene & -9.214 & 0.014 & 204.36 \\
1,3-Disopropylbenzene & -9.252 & 0.232 & 162.27 \\
1,4-Dinitobenzene & -11.340 & 0 & 168.11 \\
3,5-Dichlorophenol & -9.537 & 1.408 & 163 \\
4-Chlorophenol & -9.125 & 1.478 & 128.56 \\
4-Nitrobenzoic acid & -10.900 & 1.586 & 220.01 \\
4-Iodophenol & -9.244 & 3.313 & 119.12 \\
Methyl phenyl ether & -9.005 & 1.249 & 108.14 \\
Benzamide & -9.941 & 3.583 & 121.14 \\
Benzene & -9.653 & 0 & 78.11 \\
Chlorobenzene & -9.561 & 1.307 & 112.56 \\
Cyclohexanone & -10.310 & 2.972 & 98.14 \\
Dibenzothiophene & -8.202 & 0.524 & 184.26 \\
Phenol & -9.115 & 1.233 & 94.11 \\
Hexylbenzene & -9.299 & 0.351 & 162.27 \\
Hexachlorobutadiene & -9.444 & 0.001 & 260.76 \\
Indazole & -8.866 & 1.546 & 118.14 \\
Caffeine & -8.945 & 3.708 & 194.19 \\
Naphthalene & -8.711 & 0 & 128.17 \\
Toluene & -9.330 & 0.263 & 92.14 \\
4-Cyanophenol & -9.510 & 3.313 & 119.12 \\
\hline
\end{tabular}

analysis with the retention parameters are listed in Table.1.

\subsection{Statistical analysis}

Calculations employing the statgraphics package were run on a personal computer.

Initially, a set of 21 structural descriptors of empirical and calculation chemistry origin was subjected to a principal component analysis. The descriptors were identified which were of relevance for the prediction of retention and which at the same time have not been strongly mutually intercorrelated. Next, the stepwise regression and the multiple regression analysis were carried out keeping the requirements of the meaningful statistic in mind [26].

\section{Results and discussion}

High resolution spectra for unmodified and amine functionalized SWCNTs were determined. Samples were degassed overnight within the XPS chamber $\left(10^{-3} \mathrm{mbar}\right)$ prior to analysis of the sample. The raw XPS data for each sample were analyzed to determine peak locations and areas in relation to specific binding energies which best fit the experimental data. For the unmodified CNTs, the main $\mathrm{C}-\mathrm{C}$ peak was at $284.6 \mathrm{eV}$. For the functionalized SWCNTs, the XPS results show a broadening of the main $\mathrm{C} 1 \mathrm{~s}$ peak at $284.7 \mathrm{eV}$ as well as an additional binding energy peak at $285.8 \mathrm{eV}$ which is $1.1 \mathrm{eV}$ higher than the main $\mathrm{C} 1 \mathrm{~s}$ peak and is assigned to an amine group (C-N).
Table 2. Evaluation of retention factor for column to column, reproducibility ${ }^{\mathrm{a})}$ (CNT column)

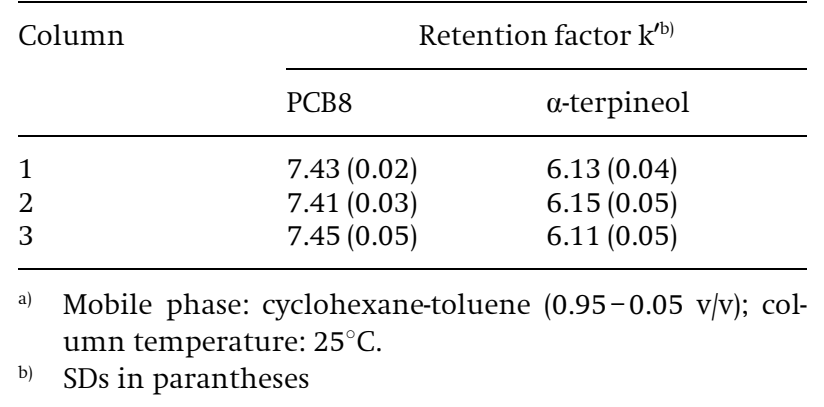

The amine functionalization is further confirmed in the N1s spectrum which presented a binding energy peak at $400.4 \mathrm{eV}$ (N1s binding energies for amines are expected between 399.5 and $400.5 \mathrm{eV}$. No N1s peak were observed in the pure CNT samples [27]. For the CNT stationary phase, the maximum CNT surface concentration determined from the carbon content was $2.4 \mathrm{mg} / \mathrm{m}^{2}$ (the percentage of surface coverage was $65 \%$ ). The maximum relative difference of the surface coverage between the four different measurements was always $0.3 \%$ for all the columns created, making a homogeneous CNT distribution in the column from the ends to the core.

To evaluate the column to column reproducibility, three CNT columns were prepared under identical conditions. The retention factors were obtained with PCB8 and $\alpha$-terpineol used as tested analytes (Table 2). The mobile phase was a cyclohexane-toluene mixture $(0.95: 0.05 \mathrm{v} / \mathrm{v})$ and the column temperature was maintained equal to $25^{\circ} \mathrm{C}$. The results showed that the technique was reliable and reproducible. In addition, typical reproducibility of this CNT column in retention time measured as RSD was $-0.5 \%$. After half a year and more than 50 times injections, the decrease for the values of retention factor on this column was $-1.2 \%$. Table 3 gave an example of the chromatographic characteristics of CNT and AP-silica columns for four solute molecules. For all analytes, the CNT column possessed a bigger retention factor. Furthermore, only on the CNT column was complete resolution attained. The separations on the CNT column for a mixture of PCBs and for another mixture containing the terpene molecules are shown in Fig. $2 \mathrm{~A}$ and $\mathrm{B}$ for a cyclohexane-toluene mixture $0.95-0.05 \mathrm{v} / \mathrm{v}$ mobile phase.

The non-covalent interaction established between the analyte and the nanomaterial includes dipole-dipole, hydrogen bonds, $\pi-\pi$ stacking, dispersion forces, dative bonds, hydrophobic and steric effect. The combination of two or more similar interactions increases the selectivity and the stability of the system. For PCBs, chlorine susbstituents in the ortho position acted as factors governing the analyte degree association with the CNT stationary phase. The retention factor obtained was in the sequence PCB1 < $\mathrm{PCB} 2<\mathrm{PCB} 3<\mathrm{PCB} 4<\mathrm{PCB} 5<$ PCB6 < 
Table 3. Characteristics for AP and CNT columns in HPLC (toluene fraction $0.05 \mathrm{v} / \mathrm{v}$ )

\begin{tabular}{lllll}
\hline Column & Tested compound & Retention factor & $\begin{array}{l}\text { Column efficiency } \\
\text { (plate number) }\end{array}$ & Resolution \\
\hline CNT & PCB5 & 3.81 & 40470 & \\
& PCB6 & 3.91 & 39998 & 1.16 (between PCB5-PCB6) \\
& linalool & 4.80 & 30068 & \\
thymol & 4.90 & 29853 & \\
AP & PCB5 & 1.19 & $*$ & $\cong 0.00$ (between linalool-thymol) \\
& linalool & 1.22 & $*$ & \\
& thymol & 1.22 & & \\
\hline
\end{tabular}

a) * not determined

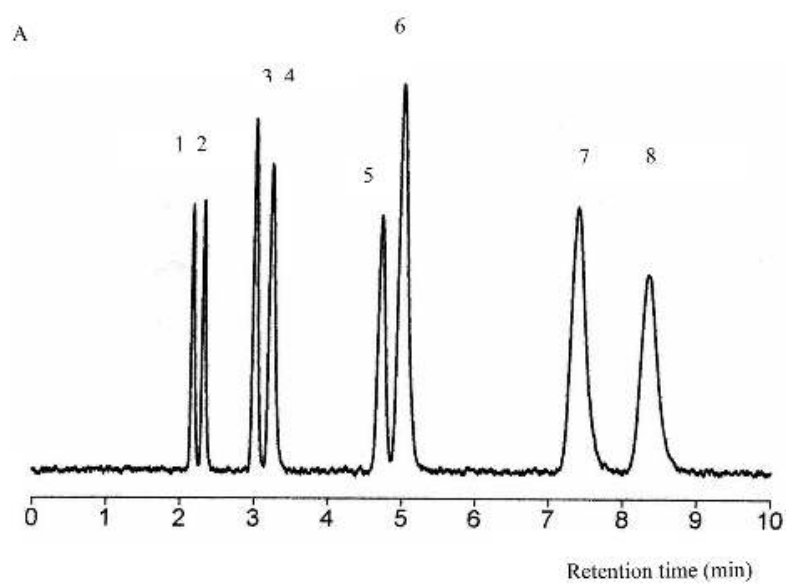

B

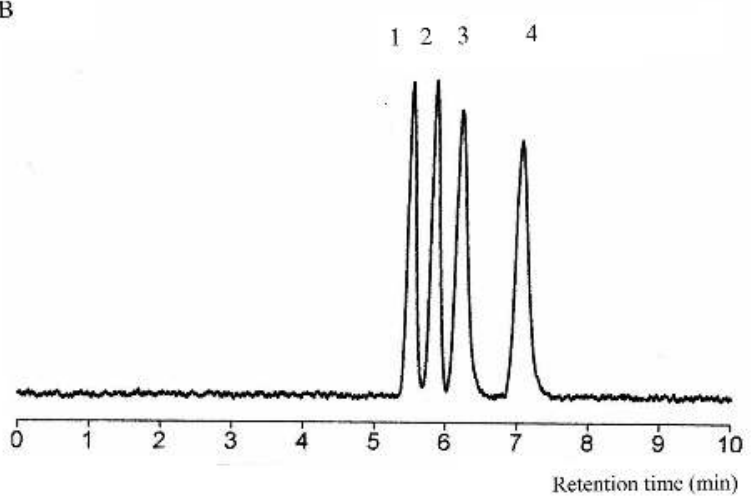

Figure 2. Separation of $P C B s(A)$ (peak $i=P C B i$ ) and terpenes (B) (peak 1, 2,3 and 4 are respectively linalool, thymol, geraniol and $\alpha$-terpineol) for a toluene-cyclohexane $(0.05-0.95 \mathrm{v} / \mathrm{v})$ mobile phase.

PCB7 < PCB8 (see Fig. 2A). PCBs with low numbers of chlorine atoms in the ortho position can more readily assume a planar conformation. Thus the CNT stationary phase shows increased affinity with increasing planarity of the PCB molecule. An increase in the retention of PCB congeners with low degrees of substitution in the ortho position has also been observed using GC on stationary phases with polar functional groups, e.g. cyano propyl groups, phenyl groups, or carborane groups [28]. The strong retention on planar PCB congeners could be caused by charge transfer interactions, as on fullerene stationary phase, or by dipole interactions. For the four terpene molecules, the retention order is linalool $<$ thymol < geraniol $<\alpha$-terpineol (Fig. 2B). Comparing the elution order between linalool and geraniol it was clearly demonstrated that the hydroxyl position in these molecules plays a major role in the retention process. For geraniol, the -OH functional group positioned in a terminal position allows the molecule to have a best fit on the CNT surface, in comparison to linalool where the -OH group is in the middle (Fig. 1). For thymol and $\alpha$-terpineol, which were cyclic molecules, thymol was eluted first. Thymol is rigid because of the aromatic ring and a non-planar geometry due to methyl substituents. Thus, these results demonstrated clearly, for these two molecule series, that a planar conformation is essential for the retention on CNT stationary phase. The influence of the mobile phase composition on the retention behavior of PCBs and terpenes was studied for a column temperature equal to $25^{\circ} \mathrm{C}$. The variation of the $\mathrm{k}^{\prime}$ values versus the toluene fraction $\mathrm{x}$ in the mobile phase was similar for PCBs and terpenes. An example of plot for PCB8 and $\alpha$-terpineol was given in Fig. 3 when the toluene fraction $\mathrm{x}$ in the mobile phase increased from 0.05 to $0.70 \mathrm{v} / \mathrm{v}$. Looking at the experimental data, it is evident that the trend is not linear. This is highlighted by the following quadratic function (Eq. 1):

$\ln k^{\prime}=\ln k^{\prime 0}-\lambda_{1} x+\lambda_{2} x^{2}$

where $k^{\prime 0}$ is the retention factor extrapolated at $\mathrm{x}=0$ and $\lambda_{1(2)}$ were constants related to the nature of the organic modifier and to the structure of the solute and the stationary phase. By using non-linear regression, for each solute molecule, the non-linear regression coefficients of Eq. (1) were determined $\left(r^{2}>0.988\right)$. From the full regression model, a Student $t$-test was used to provide the basis for the decision about whether or not the model coefficients were significant. Results of the Student's t-test show that no variables can be excluded from the model. These results confirmed that the linear solvent strength model [29-31] cannot be applied in a broad range of 


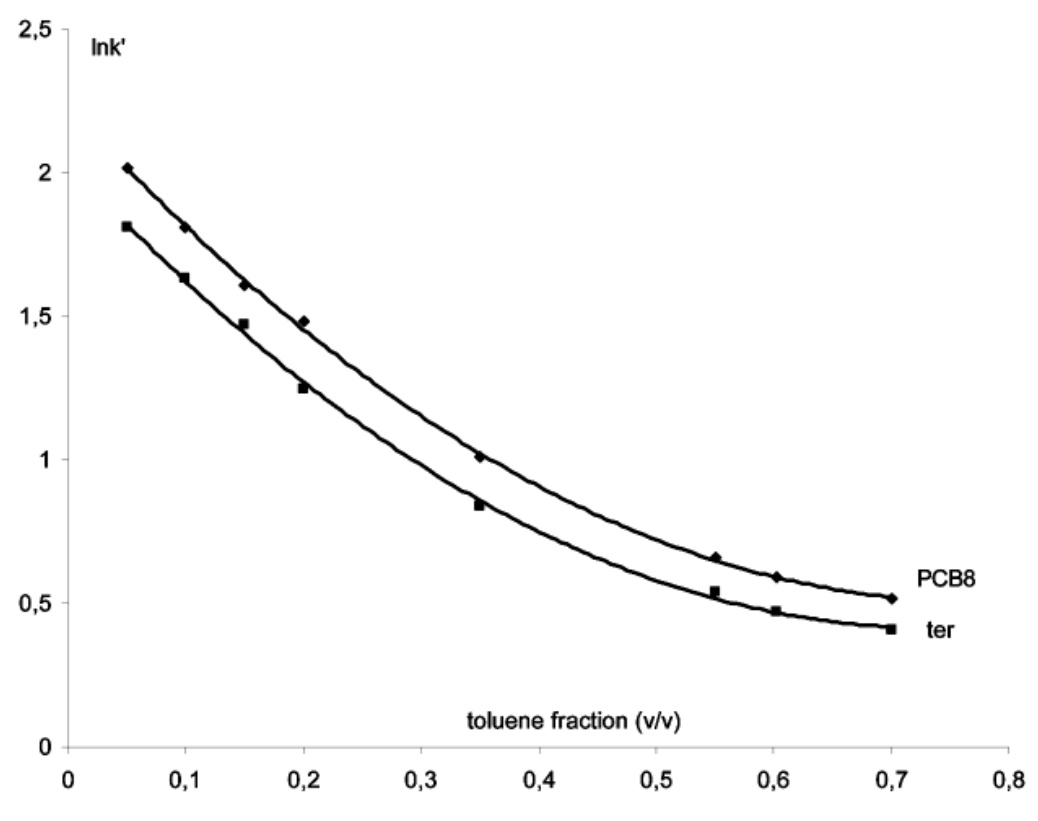

Figure 3. Logarithm retention factors for PCB8 and $\alpha$-terpineol against the volume fraction of toluene in the mixture toluenecyclohexane used as mobile phase. The column temperature was equal to $25^{\circ} \mathrm{C}$.

mobile phase compositions. The lines shown in Fig. 3 were obtained by fitting the experimental data to the quadratic function of the toluene fraction and confirmed the excellent agreement between the experimental data and the second-order relationship between $\ln k^{\prime}$ and $\mathrm{x}$. The rapid variation of the retention factors with the toluene fraction illustrates the importance of a careful optimization of the parameters of separation. The chromatograms obtained for $\mathrm{x}=0.05$ are given in Figs. $2 \mathrm{~A}$ and $\mathrm{B}$. The corresponding analysis time given by the PCB8 retention time is around 9 min for the PCBs mixture and $7 \mathrm{~min}$ for the terpenes mixture. The chromatograms obtained for $\mathrm{x}=0.35$ were given in Figs. $4 \mathrm{~A}$ and $\mathrm{B}$. The following equation were derived describing the retention parameters determined on the AP and CNT stationary phases studied in dependence on the calculated structural parameters (Eq. 2):

$\log k^{\prime}=d+m M+u \mu+E_{\text {номо }}$

where $\mu$ is the dipole moment, $\mathrm{M}$ is the molecular mass of solute and $\mathrm{E}_{\text {номо }}$ is the energy of the highest occupied molecular orbital. The measurement of retention factors of a number of solutes which descriptors ( $\left.\mathrm{M}, \mu, \mathrm{E}_{\text {номо }}\right)$ are known, in a given chromatographic system, allows the determination of the factors $(\mathrm{m}, \mathrm{u}, \mathrm{o})$ taking part in the chromatographic retention. The d intercept doest not reflect any interaction. This coefficient involves various parameters affecting retention that are not expressed by regression coefficients. The $(\mathrm{m}, \mathrm{u}, \mathrm{o})$ coefficients reflect the difference of molecular interactions in a specific system, i.e. for the given column and mobile phase composition. Since Eq. (2) is applied to the distribution between the two phases, the coefficients refer to differences in the

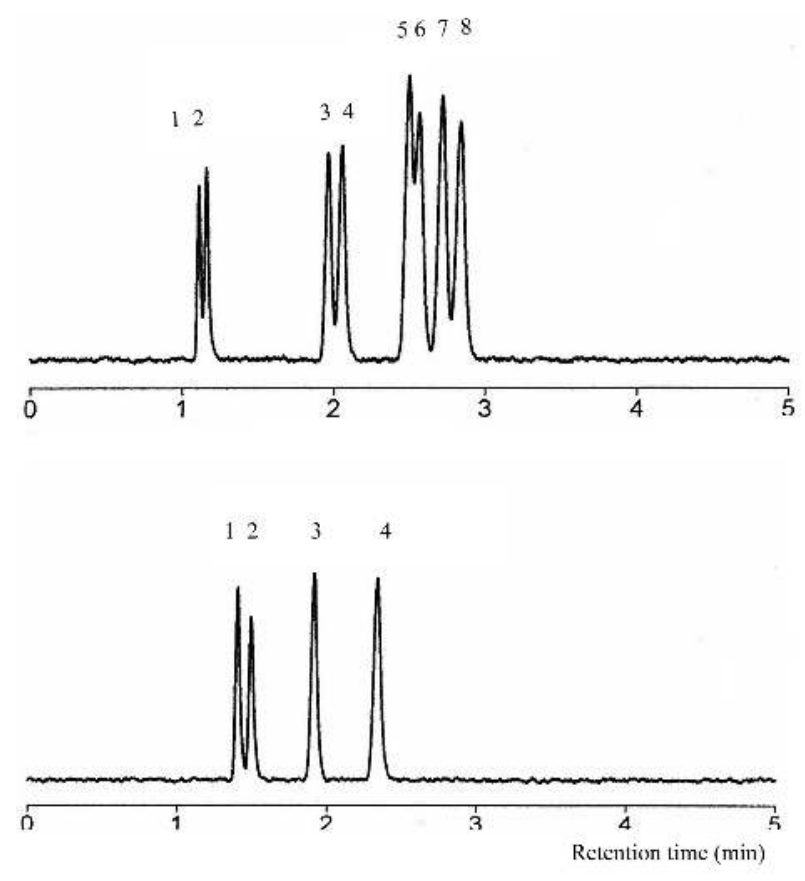

Figure 4. Separation of $P C B s A$ : (peak $i=P C B i)$ and terpenes B: (peak 1, 2, 3 and 4 are respectively linalool, thymol, geraniol and $\alpha$-terpineol) for a toluene-cyclohexane (0.35$0.65 \mathrm{v} / \mathrm{v}$ ) mobile phase.

properties between the stationary phase and the mobile phase. Thus the sign of the coefficients indicates which phase mobile or stationary phase displays the strongest interactions with the solute: if the sign is positive it indicates that the stationary phase develops the major interactions with the solute; if the sign is negative it is the mobile phase. The regression coefficients of the optimal 
Table 4. Regression coefficients (m, u, o) of the optimal model Eq. $2( \pm 95 \%$ confidence interval) and statistical parameters of individual equations for the CNT phase.

\begin{tabular}{lllllllllll}
\hline $\mathrm{x}(\mathrm{v} / \mathrm{v})$ & $\mathrm{m}$ & $95 \% \mathrm{CI}$ & $p^{\mathrm{a})}$ & $\mathrm{u}$ & $95 \% \mathrm{CI}$ & $p^{\mathrm{a})}$ & $\mathrm{o}$ & $95 \% \mathrm{CI}$ & $p^{\mathrm{a})}$ & $\mathrm{R}^{\mathrm{b})}$ \\
\hline 0.05 & 0.0208 & 0.48 & 0.0016 & 0.506 & 0.56 & 0.016 & 0.892 & 0.54 & 0.087 & 0.950 \\
0.35 & 0.0190 & 0.54 & 0.0006 & 0.289 & 0.58 & 0.014 & 0.602 & 0.59 & 0.089 & 0.980 \\
\hline
\end{tabular}

a) Statistical $p$-value.

b) Correlation coefficient.

Table 5. Regression coefficients ( $\mathrm{m}, \mathrm{u}, \mathrm{o})$ of the optimal model Eq. (2) ( $\pm 95 \%$ confidence interval) and statistical parameters of individual equations for the AP phase.

\begin{tabular}{lllllllllll}
\hline $\mathrm{x}(\mathrm{v} / \mathrm{v})$ & $\mathrm{m}$ & $95 \% \mathrm{CI}$ & $p^{\mathrm{a})}$ & $\mathrm{u}$ & $95 \% \mathrm{CI}$ & $p^{\mathrm{a})}$ & $\mathrm{o}$ & $95 \% \mathrm{CI}$ & $p^{\mathrm{a})}$ & $\mathrm{R}^{\mathrm{b})}$ \\
\hline 0.05 & 0.0112 & 0.47 & 0.0014 & 0.101 & 0.50 & 0.011 & 0.080 & 0.48 & 0.009 & 0.890 \\
0.35 & 0.0097 & 0.41 & 0.0003 & -0.081 & 0.51 & 0.009 & 0.021 & 0.40 & 0.008 & 0.830 \\
\hline
\end{tabular}

a) Statistical $p$-value.

b) Correlation coefficient.

model, i.e. $(\mathrm{m}, \mathrm{u}, \mathrm{o})$ are shown in Tables 4 and 5 for two toluene fractions in the mobile phase $(\mathrm{x}=0.05$ and $\mathrm{x}=0.35$ ) and for the CNT and AP stationary phases. The prediction of Eq. (2) is diverse and limited. The different Eq. (2) obtained gives only general trends in retention and gives reasonable retention prediction. Plots of the experimental $\log \mathrm{k}^{\prime}$ against calculated $\log \mathrm{k}^{\prime}$ values for these two $\mathrm{x}$ values show no serious outliers for the CNT stationary phase thereby indicating that the LFER model correlates with experimental results. The equations of the linear regressions were as follows: (i) for the AP stationary phase $\log \mathrm{k}_{\text {calc }}^{\prime}=0.03+0.90 \log \mathrm{k}_{\text {exp }}^{\prime}, \mathrm{R}=0.89, \mathrm{SD}=$ 0.25 for $\mathrm{x}=0.05$ and $\log \mathrm{k}_{\text {calc }}^{\prime}=0.04+0.89 \log \mathrm{k}_{\text {exp }}, \mathrm{R}=0.83$, $\mathrm{SD}=0.30$ for $\mathrm{x}=0.35$, (ii) for the CNT stationary phase $\log \mathrm{k}_{\text {calc }}^{\prime}=0.02+0.95 \log \mathrm{k}_{\text {exp }}^{\prime}, \mathrm{R}=0.95, \mathrm{SD}=0.12$ for $\mathrm{x}=$ 0.05 and $\log \mathrm{k}_{\text {calc }}^{\prime}=0.01+0.97 \log \mathrm{k}_{\text {exp }}, \mathrm{R}=0.98, \mathrm{SD}=0.11$ for $\mathrm{x}=0.35$. From these data, evidently, molecular mass of the solute plays the role of a structural descriptor of their ability to participate in the dispersive attraction interactions with both the mobile and the stationary phase molecules. Dipole moment $\mu$ of solutes can certainly be treated as a descriptor for their potency to undergo the polar intermolecular interaction of a dipole-dipole or a dipole-induced dipole type with both the eluent and the stationary phase molecules.

For $\mathrm{x}=0.05$, as the effect of this interaction on retention is positive (positive $u$ value, Tables 4 and 5) for the CNT and AP phases, it confirms that the CNT stationary phase develops these interactions principally with the solute. The u-value is dominant in the case of the CNTphase (Tables 4 and 5). This highest u-value, compared with the AP phase reflects the strongest polar interactions of solutes with the CNT stationary phase. This can explain the highest retention and resolution values obtained with the CNT-phase. This increase in the polar interactions is related to the CNT structure which can be viewed as graphite sheet $\left(\mathrm{sp}^{2}\right)$ carbon that has been rolled up into a tiny tube. There is thus growing evidence to suggest that polar interactions have a significant influence on retention. The mechanism of this effect must be elucidated, but it is possibly due to $\pi-\pi$ stacking interactions between aromatic solutes and the single walled CNTs of the CNT phase. A large contact area between them would be favorable for $\pi-\pi$ stacking. The solute adsorbed on to the CNT surface would be expected to be closer to the surface than they would be in relation to the AP surface. Since the polar interactions between atoms is proportional to $1 / \mathrm{r}^{\mathrm{n}}(\mathrm{n}>1)$ where $\mathrm{r}$ is the distance between atoms, a small decrease in $\mathrm{r}$ will greatly increase the energy of interaction. Based on this explanation, a greater retention would be expected on the CNT stationary phase. As well, since the nanotubes are full of $\pi$ electrons the aromatic ring of a solute molecule is likely to interact with the surface of tubes through $=\mathrm{CH}-\pi$ hydrogen bonds as previously demonstrated for polycyclic aromatic hydrocarbons [17]. An interesting feature of Eq. (2) and Tables 4 and 5 is the presence of the $E_{\text {номо }}$ term. o is dominant in the case of the CNT stationary phase. The value of o was for the CNT stationary phase 0.892 while the one for the AP phase was around 0.080 (Tables 4 and 5). This proves that, in this CNT phase, the electron pair donor-electron pair acceptor (charged transfer) and dipole-dipole interactions affect the HPLC separation and are developed principally between the CNT stationary phase and the solute molecule (positive o value). These results provide an objective argument that structurally specific intermolecular interactions play a great role on the molecular mechanism of retention on 
the CNT stationary phase. The adsorption and nanoscale interactions of SWCNT can largely enhance the recognition of CNT stationary phase.

The correlations were greatly affected by the fraction of toluene in the mobile phase. The strong decrease of the $(\mathrm{m}, \mathrm{u}, \mathrm{o})$ coefficients obtained for $\mathrm{x}=0.35$ (Tables 4 and 5) was explained by an increase of the dipole-dipole interaction between the solute molecule and the mobile phase (i.e. toluene) and lead to a decrease of the solute retention (Fig. 3). The negative $u$ coefficient obtained for the AP phase indicated well that this type of interaction of solutes is preferred in the mobile phase for this higher toluene fraction in the mobile phase $(0.35$ instead of $0.05)$.

In summary, chemometric processing of the HPLC data collected for an appropriately designed set of solutes allows an objective, quantitative evaluation of the CNT stationary phase. In view of that approach, the newly obtained CNT stationary phase appears distinctive from the other stationary phase with regard to its separation mechanism. The CNT stationary phase was demonstrated to provide significant input to retention and resolution due to the selective dipole-dipole and charge transfer interactions with solute molecule. Good physicochemical parameters and the specific retention properties encourage further studies on the preparation and application of this CNT phase.

The authors declared no conflict of interest.

\section{References}

[1] Valcarcel, M., Cardenas, S., Simonet, B. M., Anal. Chem. 2007, 79, 4788- 4797.

[2] Merkoci, A., Mikrochim. Acta 2006, 152, 157-174.

[3] Costa-Fernadez, M., Peireiro, R., Sanz-Medel, A., Trends. Anal. Chem. 2006, 25, 207-218.

[4] Raty, J. Y., Galli, G., J. Electroanal. Chem. (Lausanne Switz) 2005, $584,9-12$.

[5] Jiang, L., Sun, W., Weng, J., Shen, Z., Polymer 2002, 43, 15631566.
[6] Roy, D., Chowalla, M., Wang, H., Sano, N. et al., Chem. Phys. Lett. 2003, 373, 52-56.

[7] Yang, Y., Gupta, M. C., Dudley, K. L., Lawrence, R. W., Nanotechnology 2004, 15, 1545-1548.

[8] Sano, M., Kamino, A., Okamura, J., Shinkai, S., Science 2001, 293, 1299-1301.

[9] Pan, H., Liu, L., Guo, Z. X., Dai, L. et al., Nano Lett. 2003, 3, 29-32.

[10] Quay, C. H. L., Cumings, J., Gamble, S. J., Yazdani, A. et al., Compos. Sci. Technol. 2007, 67, 2965-2972.

[11] Wang, S., Liang, Z., Liu, T., Wang, B., Zhang, C., Nanotechnology 2006, 17, 1994-1998.

[12] Li, Q., Yuan, D., J. Chromatogr. A 2003, 1003, 203-209.

[13] Saridara, C., Mitra, S., Anal. Chem. 2005, 77, 7094-7097.

[14] Karwa, M., Mitra, S., Anal. Chem. 2006, 78, 2064- 2070.

[15] Li, Y., Chen, R., Xiang, D., Ciuparu, D. et al., Anal. Chem. 2005, 77, 1398-1406.

[16] Menna, E., Della negra, F., Prato, M., Tagmatarchis, N. et al., Carbon NY 2006, 44, 1609-1613.

[17] Chang, Y. X., Zhou, L. L., Li, G. X., Li, L., Yuan, L. M., J. Liq. Chromatogr. Relat. Technol. 2007, 30, 2953-2958.

[18] Sombra, L., Moliner-Martinez, Y., Cardenas, S., Valcarcel, M., Electrophoresis 2008, 29, 3850-3867.

[19] Abraham, M. H., Chem. Soc. Rev. 1993, 22, 73-83.

[20] Abraham, M. H., McGowan, J. C., Chromatographia 1987, 23, 243 248.

[21] Kaliszan, R., Quantitative Structure Chromatographic Retention Relationships, Wiley, New York 1987.

[22] Chen, J., Hamon, M., Hu, H., Chen, Y. et al., Science 1998, 282, 95 98.

[23] Liu, J., Rinzler, A. G., Dai, H. J., Hafner, J. H. et al., Science 1998, 280, $1253-1256$.

[24] Ramanathan, T., Fisher, F. T., Ruoff, R. S., Brinson, L. C., Chem. Mater. 2005, 17, 1290-1295.

[25] Buszewski, B., Gilpin, R. K., Jaroniec, M., J. Chromatogr. 1994, 673, 11-19.

[26] Charton, M., Clementi, S., Ehrenson, S., Exner, O. et al., Quant. Struct. -Act. Relat. 1985, 4, 29-30.

[27] Moulder, J. F., Stickle, W. F., Sobol, P. E., Bomben, K. D., Handbook of X-ray photoelectron spectroscopy, Perkin-Elmer Corporation, Eden Prairie, MN 1992.

[28] Larsen, B., J. High. Resolut. Chrom. 1995, 18, 141-151.

[29] Snyder, L. R., Kirkland, J. J., Introduction to Modern Liquid Chromatography, John Wiley \& Sons, New York 1979.

[30] Snyder, L. R., Stadalius, M. A., High Performance Liquid Chromatography: Advances and Perspectives, Academic Press, New York 1986.

[31] Jandera, P., Churacek, J., Gradient elution in column liquid chromatography: Theory and Practice, Elsevier, Amsterdam 1985. 